\title{
DELIMITAÇÃO DE SÍTIOS FLORESTAIS E ANÁLISE DOS FRAGMENTOS PERTENCENTES NA BACIA DO RIO ITAPEMIRIM
}

\author{
FORESTRY SITES DELIMITATION AND ANALYSIS OF FOREST \\ FRAGMENTS AT ITAPEMIRIM RIVER WATERSHED
}

Yhasmin Gabriel Paiva ${ }^{1}$; Kennedy Ribeiro da Silva ${ }^{1}$; José Eduardo Macedo Pezzopane ${ }^{2}$; André Quintão de Almeida ${ }^{3}$; Roberto Avelino Cecílio ${ }^{4}$

\section{RESUMO}

\begin{abstract}
Devido às características ambientais e sócio-econômicas, a Bacia do Rio Itapemirim (BRI) apresenta grande potencial para o desenvolvimento de empreendimentos florestais com objetivos econômicos e ambientais. Este trabalho consiste em delimitar sítios florestais na BRI, a partir de parâmetros climáticos, edáficos e topográficos e verificar a condição atual da cobertura florestal na BRI. Para delimitação dos sítios florestais foram utilizados dados SRTM de elevação, mapa das classes de solos da bacia e a caracterização da disponibilidade hídrica a partir das Equações de Thorntwaite \& Matter (1955). Os fragmentos florestais foram digitalizados através da classificação visual das imagens do satélite CBERS-CCD. Verificou-se que os parâmetros utilizados apresentaram bons resultados para a delimitação dos sítios florestais na BRI. O diagnóstico da condição atual da cobertura florestal na Bacia do Rio Itapemirim foi importante para o estudo mais detalhado em relação à cobertura florestal presente em cada sítio delimitado.
\end{abstract}

Palavras-chave: Floresta Atlântica, dados SRTM, CBERS-CCD

\begin{abstract}
Itapemirim River Watershed (BRI) brings a great potential to the development of forestry undertakings due to its environmental, social and economics characteristics. This paper shows the delimitation of forestry sites at BRI accounting for climactic, soil and topographical data. A study of the Atlantic Forest fragments conditions at BRI was also done. Forestry sites were delimitated using SRTM elevation data, Thorntwaite \& Matter water balance and soil classes maps. Atlantic Forest fragments were individualized by visual classification of CBERS-CCD satellite images. Results showed that the use of climatic, soil and topographical data are very helpful in forestry sites delimitation.
\end{abstract}

Key words: Atlantic Forest, SRTM date, CBERS-CCD

\section{INTRODUÇÃO}

A delimitação de áreas homogêneas constitui uma eficiente ferramenta no planejamento do uso de recursos naturais e na otimização de investimentos. Esta prática permite avaliar a capacidade produtiva de uma determinada área, correlacionando com a espécie ou espécies que nela se deseja produzir.
Desta forma, grande parte das decisões tomadas no manejo florestal tem base mapeamentos de Habitats Florestais (Andrade, 1998).

Considerados como de maior influência na distribuição e no desenvolvimento da vegetação, os fatores climáticos são os principais componentes em estudos de classificação e zoneamento (Neto, 2000). A disponibilidade hídrica dos solos

\footnotetext{
Engenheiro Florestal, Universidade Federal do Espírito Santo CCA-UFES, CEP 29500-000 Alegre-ES. yhasminp@ hotmail.com Engenheiro Florestal, DS, Professor Adjunto, Universidade Federal do Espírito Santo, CCA-UFES, CEP 29500-000 Alegre-ES.

Engenheiro Florestal, Mestre em Engenharia Ambiental, Universidade Federal do Espírito Santo. CT-UFES, CEP: 29060-900 Vitória-ES, CT-UFES.

4 Engenheiro Agrícola, DS, Professor Adjunto, Universidade Federal do Espírito Santo, CCA-UFES, CEP 29500-000 Alegre-ES.
} 
é influenciada pelas condições térmicas e pela distribuição espaço temporal das precipitações. A temperatura influencia os processos fisiológicos da planta, como fotossíntese, respiração, transpiração e divisão celular; enquanto a água é fundamental a todos os processos fisiológicos e bioquímicos (Pereira et al., 2002).

Segundo a Fundação SOS Mata Atlântica e INPE (2002), restam atualmente apenas $8 \%$ da área originalmente coberta pela Mata Atlântica. Esta se encontra extremamente fragmentada, o que torna este ecossistema frágil, despertando preocupações e evidenciando a necessidade de estudos visando subsidiar ações para manter a sustentabilidade dos fragmentos. Para que tornem possíveis as ações que promovam a recuperação e até mesmo a interligação dos fragmentos florestais, através dos corredores ecológicos, é necessária a realização do diagnóstico da atual situação.

Devido às características ambientais e sócioeconômicas a Bacia do Rio Itapemirim (BRI) apresenta um grande potencial para o desenvolvimento de empreendimentos florestais com objetivos econômicos e ambientais. No entanto, a inexistência de uma caracterização ambiental em escala adequada para a bacia e a ausência de trabalhos científicos que indiquem as áreas mais adequadas para o plantio de espécies arbóreas podem comprometer o sucesso dos projetos florestais na região.

O objetivo deste trabalho é delimitar áreas internamente homogêneas e heterogêneas entre si, denominadas sítios florestais, levando-se em consideração fatores edáficos, de relevo e climáticos, realizando posteriormente o diagnóstico da condição atual da cobertura florestal na BRI em relação aos sítios estabelecidos.

\section{MATERIAL E MÉTODOS}

A área de estudo compreende a bacia hidrográfica do Rio Itapemirim, localizada na região Sul do Estado do Espírito Santo, com aproximadamente 592176 ha. A bacia situa-se entre os meridianos $40^{\circ} 48^{\prime}$ 'e $41^{\circ} 52$ ' de longitude Oeste e entre os paralelos $20^{\circ} 10^{\prime}$ e $21^{\circ} 15^{\prime}$ de latitude Sul, abrangendo 17 municípios.

Para a delimitação dos sítios florestais foram utilizados parâmetros edáficos; a altitude como fator topográfico; dados climáticos representados pela temperatura, precipitação, déficit e excedente hídrico e relação de evapotranspiração real e potencial.

As classes de solos da bacia foram obtidas por meio da digitalização da carta do Estado do Espírito Santo, produzida pela Empresa Brasileira de Pesquisa Agropecuária (EMBRAPA, 1999) realizada na escala 1:400.000.

O modelo digital de elevação do terreno utilizado foi originado dos dados de altitude disponibilizados pelo projeto SRTM (Shuttle Radar Topography Mission), obtidos de Miranda e Coutinho (2004). Tais dados apresentam resolução espacial de $90 \mathrm{~m}$ e escalas de digitalização de 1: 250.000.

As temperaturas do ar foram estimadas por meio do modelo matemático desenvolvido por Pezzopane et al. (2004) para o estado do Espírito Santo, no qual a latitude e a altitude são as variáveis independentes e a temperatura do ar a variável dependente, como demonstrado a seguir:

$$
\mathrm{Y}_{\mathrm{i}}=\beta_{0}+\beta_{1} \text { Alt }+\beta_{2} \text { Lat }
$$

em que:

$\mathrm{Y}_{\mathrm{i}}=$ Temperatura do $\operatorname{ar}\left({ }^{\circ} \mathrm{C}\right)$;

Alt $=$ Altitude $(\mathrm{m})$;

Lat $=$ Latitude, em graus e décimos;

$\beta_{0}, \beta_{1}$ e $\beta_{2}=$ coeficientes da equação de regressão ajustados para cada mês do ano.

Na caracterização da disponibilidade hídrica na BRI foram utilizadas séries históricas de chuvas (30 anos) coletadas em postos pluviométricos da Agência Nacional de Águas (ANA). Utilizou-se dados de precipitação média anual, obtidos em 16 postos pluviométricos (Figura 1-a), 13 na bacia e três na vizinhança no período de 1976 a 1997. A inclusão de postos pluviométricos fora do limite da bacia teve como objetivo eliminar o efeito de borda no processo de interpolação, sendo também utilizado por Moreira (1997) e Andrade (1998).

A evapotranspiração potencial foi calculada pelo modelo proposto por Thornthwaite (1948), que é baseado na temperatura média do ar e radiação solar no topo da atmosfera.

A disponibilidade hídrica foi determinada a partir de resultados dos balanços hídricos climatológicos médios (Thornthwaite e Matther, 1955) com dados de déficit e excedente hídricos, calculados em função dos dados de chuva e evapotranspiração mensais. A partir dos valores de evapotranspiração 
real (ER) e potencial (EP) calculou-se a razão ER/ EP. Adotou-se capacidade de armazenamento de água no solo de $100 \mathrm{~mm}$.

Na espacialização dos dados climáticos (Figura 1-b), foi utilizado o software IDRISI versão 32. O método utilizado na interpolação foi o da Krigagem linear, propiciando melhores resultados e ser de mais fácil interpretação (Andrade, 1998).

Com os valores individuais de cada pixel do mapa, obtidos dos parâmetros avaliados e suas coordenadas geográficas correspondentes, os dados foram exportados para posterior análise.

Foi efetuada a análise estatística dos dados usando-se o software MATLAB 6.0. Realizou-se o agrupamento não-hierárquico, com o método convergente de agrupamento. Os resultados das análises foram reportados ao software de geo-processamento e assim delimitados os sítios ambientais homogêneos na Bacia Hidrográfica do Rio Itapemirim.

Para realizar o diagnóstico da condição atual dos fragmentos de Mata Atlântica presentes na região da bacia, utilizou-se as imagens do satélite CBERS-2 ("China-Brazil Earth Resources"), produto do sensor CCD, com resolução espacial de 20 m, obtidas através do endereço eletrônico: http:// www.dgi.inpe.br/CDSR na data 03/04/2005. Este sensor possui cinco bandas espectrais, três na faixa do visível uma no infravermelho próximo e outra na pancromática.

Foram adquiridas quatro cenas que compõem toda a áera da bacia. As imagens foram processadas nas coordendadas UTM (Universal Transversa de Mercator), zona 24, datum SAD 69. Realizou-se a correção geométrica e posteriormente o mosaico das quatro imagens com as suas respectivas bandas.

A identificação das áreas de florestas na imagem pôde ser melhor realizada através da composição nas bandas espectrais do azul, verde e infravermelho próximo.

Dispostos dos dados de cobertura florestal nativa na região da bacia, foi verificada a área total de fragmentos pertencentes a cada sítio delimitado para posterior análise.

\section{RESULTADOS E DISCUSSÃO}

Os sítios florestais delimitados na região da bacia do rio Itapemirim - BRI são expostos na Figura 2 apresentando três classes. O sítio 1 se encontra ao norte da bacia e determinadas regiões centrais, é o maior sítio delimitado com área de cerca de 343759 ha, correspondendo a $58.1 \%$ da área total da bacia. O sítio 2 possui área de 151185 ha $(25.5$ $\%$ da área total), situado ao centro da bacia. O sítio 3 , menor deles, localiza-se ao sul da bacia ocupando uma área de 97230 ha (16.4\% da área total).

Os parâmetros para a formação de sítios florestais se encontram na Tabela 1 com valores médios de cada um deles em cada sítio delimitado.

O sítio 1 apresenta altitudes médias expressivamente superior às demais, deve-se ao fato de, nesta

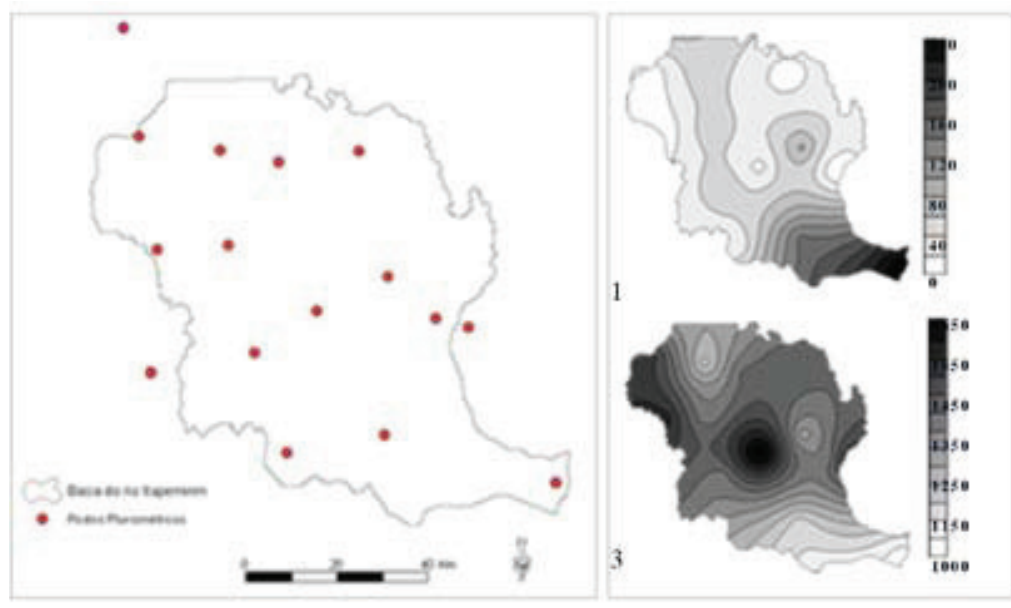

a)

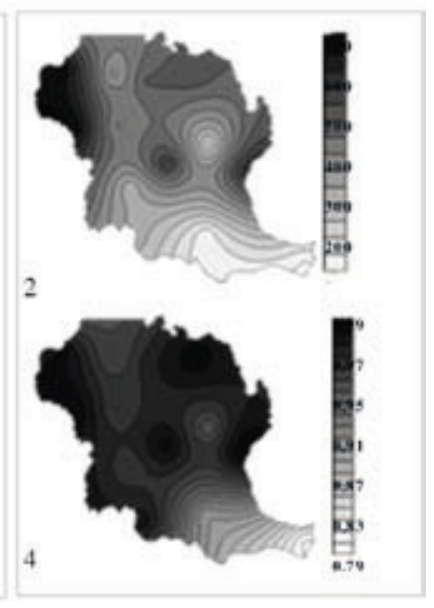

b)

Figura 1. Postos pluviométricos (a); Mapas interpolados: 1- Excedente Hídrico; 2- Déficit Hídrico; 3- Precipitação e 4- ER/EP (b). 


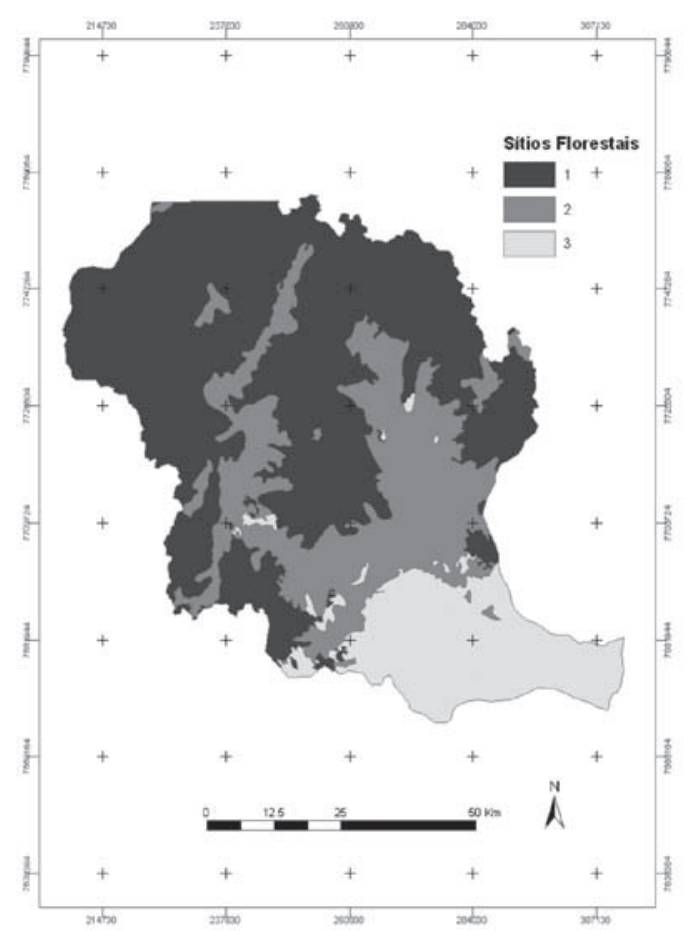

Figura 2. Sítios florestais delimitados na Bacia Hidrográfica do Rio Itapemirim.

região da Bacia, se encontrar o Parque nacional do Caparaó, onde se localiza o Pico da Bandeira com elevação de $2874 \mathrm{~m}$. Verifica-se que, neste mesmo sítio, são encontrados os menores valores médios de temperatura $\left(19,16^{\circ} \mathrm{C}\right)$. O relevo é um fator intrínseco topoclimático, que condiciona o terreno a diferentes exposições à radiação solar direta, logo a configuração do terreno também influi na sua temperatura (Pereira et al. 2002). Confirmando esta correlação, no sítio 3, que abriga o litoral, ou seja, nível de altitudes próximas a zero, a temperatura média foi a maior do que os demais $\left(23,82^{\circ} \mathrm{C}\right)$.
A temperatura do ar exerce influência sobre vários aspectos da produtividade vegetal, estando relacionada com o crescimento e desenvolvimento das plantas, devido ao seu efeito na velocidade das reações químicas e dos processos internos de transporte (Pereira et al. 2002). Antes de se realizar o plantio deve-se, portanto verificar as exigências das espécies em relação a este parâmetro combinados a outros fatores. Pode-se recomendar, para locais com menores temperaturas como, por exemplo, no sítio 1, as espécies: Araucária angustifólia, Pinus elliottii; em locais com temperaturas mais altas, espécies como Cariniana legalis, Dalbergia nigra, Cedrella fissilis dentre outras, além do gênero Eucalipto, amplamente utilizado em projetos de reflorestamento.

A quantidade e a distribuição de chuvas que ocorrem anualmente em uma região podem determinar o tipo de vegetação natural existente já que a água atua como fator indispensável no desenvolvimento da vegetação. O sítio ambiental 1 é a região que apresenta os maiores valores de precipitação anual, com média de $1414 \mathrm{~mm}$, o que pode indicar uma alta taxa de produção de biomassa. Segundo Leopoldo et al., (1981), a cobertura do terreno está intimamente ligada à área foliar, tendo menor influência à quantidade de chuva, assim, essa produção de biomassa fica também condicionado ao grau de interceptação de água pela arquitetura do dossel da floresta.

Nos sítios 2 e 3 foram encontrados valores médios da relação de evapotranspiração 0,92 e 0,87 respectivamente. Para estes, o suprimento hídrico apresenta-se mais restrito já que valores acima de 0,95 desta relação referem-se a condições de deficiencia hídrica, podendo limitar o crescimento e reprodução à cultura florestal a ser implantada, Verifica-se que no sítio 3, a condição média de déficit é mais acentuada (112 mm). Assim, devese ao realizar o plantio de povoamentos florestais, dispor de suporte de irrigação para um melhor desenvolvimento da espécie.

Tabela 1

Média dos parâmetros de climatológicos e de relevo nos sítios da BRI.

\begin{tabular}{|c|c|c|c|c|c|c|}
\hline Sítios & Precipitação $(\mathrm{mm})$ & Altitude $(\mathrm{m})$ & Deficit $(\mathrm{mm})$ & $\mathrm{Er} / \mathrm{Ep}$ & Excedente $(\mathrm{mm})$ & Temperatura $\left({ }^{\circ} \mathrm{C}\right)$ \\
\hline 1 & 1414,00 & 1926,54 & 34,00 & 0,95 & 426,00 & 19,16 \\
\hline 2 & 1385,00 & 701,07 & 47,00 & 0,92 & 336,00 & 22,48 \\
\hline 3 & 1224,00 & 180,66 & 112,00 & 0,87 & 161,00 & 23,82 \\
\hline
\end{tabular}


A Figura 3 indica a situação atual das florestas nativas na região da bacia, evidenciadas em cada sítio delimitado.

A área total de fragmentos florestais da bacia foi de cerca de 116867 ha, correspondendo a 19,7 $\%$ da área da bacia. Deve-se salientar que este resultado deve ser avaliado sabendo que a imagem CBERS-CCD é de média resolução (Nakamura e Novo, 2005), portanto, o nível de detalhamento é reduzido.

Nota-se que a cobertura florestal existente no sítio 1 é melhor conservada que os demais, 24,2 $\%$ de sua área é composta de floresta (83147 ha). Este sítio localiza-se na região serrana onde são encontradas, como já verificado, as maiores altitudes, além da topografia acidentada, tornado essas Áreas de Preservação Permanente (APP's). O sítio 2 e 3 possuem 15,3\% e 11\% de áreas de matas conservadas correspondendo a 23072,9 ha e 10647 ha respectivamente.

A floresta presente à bacia apresenta-se bastante fragmentada com 1957 fragmentos (Tabela 2). Esta intensa fragmentação é decorrente principalmente de atividades agrícolas presentes na bacia como, por exemplo, pastagens e cafeicultura iniciados historicamente com retirada de matas nativas.

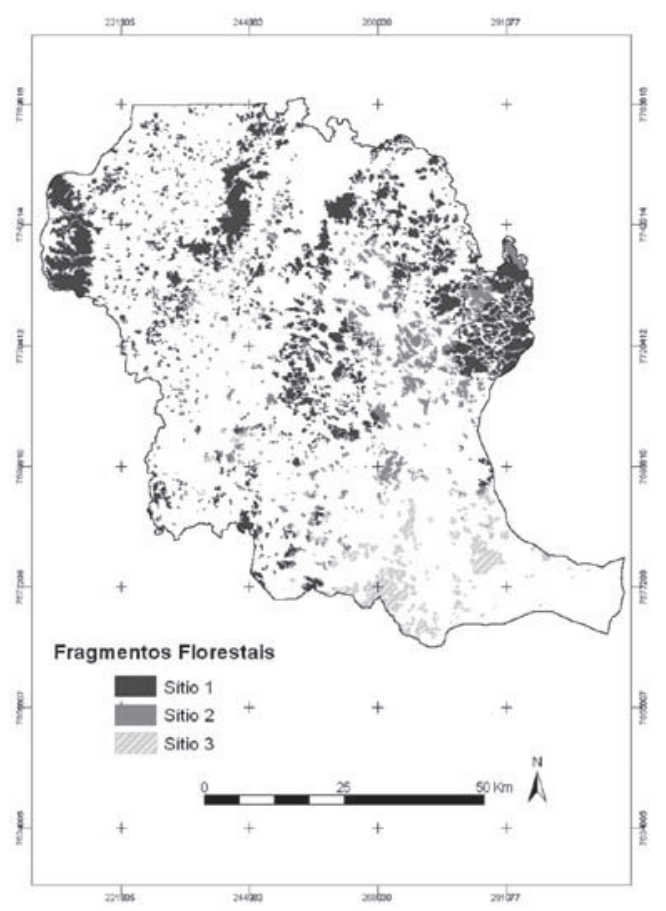

Figura 3. Fragmentação na Bacia do Rio Itapemirim.
Verifica-se que a maior parte dos fragmentos florestais da bacia se encontra na classe de área de 1 a 20 ha. Este resultado indica que cerca de metade deles possuem áreas reduzidas, estando sugeitos a maiores interferências de atividades antropogênicas, o que pode causar desequilíbrios a estes ambientes e consequente extinção destes fragmentos de mata nativa.

Com o conhecimento da situação atual das florestas naturais verificados região da bacia, viu-se necessárias implantações de reflorestamentos como alternativas ecológicas e econômicas. A delimitação dos sítios ambientais pode contribuir para posteriores estudos e aplicações como o planejamento ambiental orientando o plantio de essências florestais e ainda realização de zoneamentos de espécies florestais na bacia do Rio Itapemirim.

Tabela 2

Dados divididos em classe correspondente a área e percentuais da fragmentação florestal, encontrados através de uma imagem CBERS -2 na Bacia do Rio Itapemirim, Sul do Espírito Santo

\begin{tabular}{|c|c|c|}
\hline \multirow{2}{*}{$\begin{array}{c}\text { Classes de } \\
\text { Área (ha) }\end{array}$} & \multicolumn{2}{|c|}{ Fragmentos } \\
\cline { 2 - 3 } & $\mathbf{N}^{\mathbf{0}}$ de Ocorrência & $\%$ \\
\hline$<1$ & 69 & 3,57 \\
\hline $1-20$ & 934 & 47,72 \\
\hline $20-40$ & 353 & 18,03 \\
\hline $40-60$ & 191 & 9,75 \\
\hline $60-80$ & 90 & 4,59 \\
\hline $80-100$ & 68 & 3,47 \\
\hline$>100$ & 252 & 12,87 \\
\hline Total & 1957 & 100 \\
\hline
\end{tabular}

\section{CONCLUSÕES}

Os resultados obtidos permitem concluir que a utilização de variáveis climáticas, juntamente com componentes do balanço hídrico, classes de solos e atributos do relevo, contribuem satisfatoriamente na delimitação de sítios florestais.

O diagnóstico da condição atual da cobertura florestal na Bacia do Rio Itapemirim foi importante para o estudo mais detalhado em relação à cobertura florestal presente em cada sítio delimitado. 


\section{LITERATURA CITADA}

ANDRADE L.A. 1998. Classificação ecológica do território brasileiro situado a leste do meridiano de $44^{\circ}$ oeste e ao norte do paralelo de $16^{\circ}$ sul: uma abordagem climática. 1998. 147 p. Dissertação (Doutorado em Ciências Florestais). Universidade Federal de Viçosa, Viçosa.

EMBRAPA. 1999. Centro Nacional de Pesquisa de Solos (Rio de Janeiro, RJ). Sistema Brasileiro de classificação de solos. Brasília: Embrapa-SPI; Rio de Janeiro: Embrapa-CNPS, 1412 p.

FUNDAÇÃO SOS MATA ATLÂNTICA E INSTITUTO NACIONAL DE PESQUISAS ESPACIAIS (INPE). 2002. Atlas dos Remanescentes Florestais da Mata Atlântica: período 1995-2000. Relatório Final.

LEOPOLDO, P.R.; SOUZA, A.P.; TUACEK FILHO, S. 1981. Interceptação da água da chuva e cultura de cana-de-açucar. Brasil Açucareiro, 98(6): 9-16.

MIRANDA, E.E.; COUTINHO, A.C. (COORD.). 2007. Brasil Visto do Espaço. Campinas: Embrapa Monitoramento por Satélite, 2004. Disponível em: <http://www.cdbrasil.cnpm. embrapa.br>. Acesso em: 25 abr.

MOREIRA, I.P.S. 1997. Classificação ecológica do território brasileiro situado entre 16 e $24^{\circ}$ de latitude Sul e $39^{\circ} 51$ de longitude Oeste. 1997. 156 p. Dissertação (Doutorado em Ciências Florestais). Universidade Federal de Viçosa, Viçosa.
NAKAMURA, J.; NOVO, E. 2005. Mapeamento da mancha urbana utilizando imagens de média resolução: sensores CCD/CBERS2 e TM/Landsat5 - estudo de caso da cidade de Rio Branco-Acre. In: Simpósio Brasileiro de Sensoriamento Remoto, 12, 2005. Goiânia. Anais... Goiânia: INPE, p. 3843-3850.

NETO, S.N.O. DE. 2000. Classificação ecológica do território brasileiro localizado entre 16 e $24^{\circ}$ latitude sul e 48 e $60^{\circ}$ longitude oeste: uma abordagem climática. 2000.109 p. Dissertação (Doutorado em Ciências Florestais). Universidade Federal de Viçosa, Viçosa.

PEREIRA, A.R.; ANGELOCCI, L.R.; SENTELHAS, P.C. 2002. Agrometeorologia. Porto Alegre: Editora Agropecuária, $190 \mathrm{p}$.

PEZZOPANE, J.E.M.; SANTOS, E.; ELEUTÉRIO, M.M.; A.; REIS, E. F.; SANTOS, A. R. 2004. Espacialização da temperatura do ar no Espírito Santo. Revista Brasileira de Agrometeorologia, v. 12, n. 1, p. 151-158.

THORNTHWAITE, C.W., MATTER, J.R. 1955. The water balance. New Jersey: Publications in Climatology, 8, Centerton, New Jersey, $150 \mathrm{p}$.

THORNTHWAITE, C.W. 1948. Na approach toward a rational classification of climate. Geographical Review. 38: 55-94. 